\title{
Some applications of the extended Bendixson-Dulac Theorem
}

Armengol Gasull and Hector Giacomini

\begin{abstract}
During the last years the authors have studied the number of limit cycles of several families of planar vector fields. The common tool has been the use of an extended version of the celebrated Bendixson-Dulac Theorem. The aim of this work is to present an unified approach of some of these results, together with their corresponding proofs. We also provide several applications.
\end{abstract}

\section{The Bendixson-Dulac Theorem}

Ivar Bendixson and Henri Dulac are the fathers of the today known as BendixsonDulac Theorem. The classical version of this theorem appears in most textbooks on differential equations; see $[14,37,38]$ with many applications. Let us recall it. Consider a $\mathscr{C}^{1}$-planar differential system

$$
\dot{x}=P(x, y), \quad \dot{y}=Q(x, y),
$$

defined in some open simply connected subset $\mathscr{U} \subset \mathbb{R}^{2}$, and set $X=(P, Q)$. Assume that there exists a $\mathscr{C}^{1}$ function $D: \mathscr{U} \rightarrow \mathbb{R}$, such that

$$
\left.\operatorname{div}(D X)\right|_{\mathscr{U}}=\frac{\partial(D(x, y) P(x, y))}{\partial x}+\left.\frac{\partial(D(x, y) Q(x, y))}{\partial y}\right|_{\mathscr{U}} \geq 0 \quad(\text { or } \quad \leq 0)
$$

Armengol Gasull

Departament de Matemàtiques. Universitat Autònoma de Barcelona, Edifici C 08193 Bellaterra, Barcelona. Spain, e-mail: gasull@mat.uab.cat

Hector Giacomini

Laboratoire de Mathématiques et Physique Théorique. Faculté des Sciences et Techniques. Université de Tours, C.N.R.S. UMR 7350. 37200 Tours. France, e-mail: Hector.Giacomini@1mpt.univtours.fr 
vanishing only on a set of zero Lebesgue measure. Then system (1) has no periodic orbits contained in $\mathscr{U}$. This function $D$ is usually called a Dulac function of the system.

This theorem has been extended to multiple connected regions, see for instance $[5,17,27,36]$ obtaining then a method for determining upper bounds of the number of limit cycles in $\mathscr{U}$. In the next section we recall this extension and present the proof given in [17].

As we will see this extension can be used if it is possible to find a suitable function $V$, a real number $s$, and a domain $\mathscr{U} \subset \mathbb{R}^{2}$ such that

$$
M=\frac{\partial V}{\partial x} P+\frac{\partial V}{\partial y} Q+\left.s\left(\frac{\partial P}{\partial x}+\frac{\partial Q}{\partial y}\right) V\right|_{\mathscr{U}}
$$

does not change sign and vanishes on a set of zero Lesbesgue measure. Moreover, the upper bound given by the method for the number of limit cycles depends on the number and distribution of the ovals of $\{V(x, y)=0\}$ in $\mathscr{U}$.

When all the involved functions $P, Q$ and $V$ are polynomials this approach relates both parts of Hilbert's Sixteenth Problem. Recall that the first part deals with the number and distribution of ovals of a real algebraic curve in terms of its degree while the second part asks to find an uniform bound of the number of limit cycles of systems of the form (1) when both polynomials have a given degree; see [24, 35].

Notice that the importance of the use of the Bedixson-Dulac results is that in many cases they translate the problem of knowing the number of periodic solutions of a planar polynomial differential equation to a problem of semi-algebraic nature: the control of the sign of a polynomial in a suitable domain.

Analogously to Lyapunov functions, the first difficulty to apply these results is to find a suitable Dulac function. The problem of its existence, in the basin of attraction of critical points, is treated in [3]. A second difficulty of the method is to find a suitable region $\mathscr{U}$.

The aim of this paper is to present an unified point of view of some of the results obtained by the authors in $[17,18,19,20]$, together with some proofs. These results give methods to find Dulac functions $D$, or equivalently functions $V$ and values $s$, for which the corresponding expression $M$ is simple and so its sign can be easily studied. We also apply the method to give an upper bound of the number of limit cycles for several families of planar systems.

The Bendixson-Dulac approach has been extended in several directions: to prove non-existence of periodic orbits in higher dimensions, see [15, 26]; to control the number of isolated periodic solutions of some non-autonomous Abel differential equations, see for instance $[1,10]$; to prove non-existence of periodic orbits for some difference equations, see [29].

We can not end this introduction without talking about the contributions on the use of Dulac functions of our friend and colleague Leonid Cherkas, sadly recently deceased. His important work in this subject started many years ago and arrives until the actuality, continued by his collaborators, see for instance $[4,5,6,7,8,9,10]$ and the reference therein. In fact, one of the main motivations for the fist author to 
work in this direction were the pleasant conversations with him walking around the beautiful gardens of the Beijing University in the summer of 1990.

\subsection{The Bendixson-Dulac Theorem for multiple connected regions}

An open subset $\mathscr{U}$ of $\mathbb{R}^{2}$ with smooth boundary, is said to be $\ell$-connected if its fundamental group, $\pi_{1}(\mathscr{U})$ is $\mathbb{Z} * \cdots * \mathbb{Z}$, or in other words if $\mathscr{U}$ has $\ell$ holes. We will say that $\ell(\mathscr{U})=\ell$. We state and prove, following [17], the extension of the Bendixson-Dulac Theorem to more general domains; see other proofs in $[5,27,36]$. As usual, $\langle\cdot, \cdot\rangle$ denotes the scalar product in $\mathbb{R}^{2}$.

Extended Bendixson-Dulac Theorem. Let $\mathscr{U}$ be an $\ell$-connected open subset of $\mathbb{R}^{2}$ with smooth boundary. Let $D: \mathscr{U} \rightarrow \mathbb{R}$ be a $\mathscr{C}^{1}$ function such that

$$
M:=\operatorname{div}(D X)=\frac{\partial D}{\partial x} P+\frac{\partial D}{\partial y} Q+D\left(\frac{\partial P}{\partial x}+\frac{\partial Q}{\partial y}\right)=\langle\nabla D, X\rangle+D \operatorname{div}(X)
$$

does not change sign in $\mathscr{U}$ and vanishes only on a null measure Lebesgue set, such that $\{M=0\} \cap\{D=0\}$ does not contain periodic orbits of (1). Then the maximum number of periodic orbits of (1) contained in $\mathscr{U}$ is $\ell$. Furthermore each one of them is a hyperbolic limit cycle that does not cut $\{D=0\}$ and its stability is given by the sign of DM over it.

Proof. Observe that $\left.M\right|_{\{D=0\}}=\left.\langle\nabla D, X\rangle\right|_{\{D=0\}} \geq 0$ does not change sign in $\mathscr{U}$. Since, by hypothesis, there are no periodic orbits of (1) contained in $\{M=0\} \cap\{D=$ $0\}$, we have that the periodic orbits of (1) do not cut $\{D=0\}$.

If $\mathscr{U}$ is simply connected $(\ell=0)$ then by the Bendixson-Dulac Theorem we have that (1) has no periodic orbits in $\mathscr{U}$. We give now a proof for an arbitrary $\ell$. Assume that system (1) has $\ell+1$ different periodic orbits $\gamma_{i}$, included in $\mathscr{U}$. These orbits induce $\ell+1$ elements $\bar{\gamma}_{i}$ in the first homology group of $\mathscr{U}, H_{1}(\mathscr{U})=\mathbb{Z} \oplus \stackrel{(\ell)}{\cdots} \oplus \mathbb{Z}$. Since this group has at most $\ell$ linearly independent elements it follows that there is a non trivial linear combination of them giving $0 \in H_{1}(\mathscr{U})$. Then $\sum_{i=1}^{\ell+1} m_{i} \bar{\gamma}_{i}=0$, with $\left(m_{1}, \ldots, m_{\ell+1}\right) \neq 0$.

This last fact means that the curve $\sum_{i=1}^{\ell+1} m_{i} \gamma_{i}$ is the boundary of a two cell $C$ for which Stokes Theorem can be applied. Then

$$
\iint_{C} \operatorname{div}(D X)=\int_{\sum_{i=1}^{\ell+1} m_{i} \gamma_{i}}\langle D X, \mathbf{n}\rangle .
$$

Note that the right hand term in this equality is zero because $D X$ is tangent to the curves $\gamma_{i}$ and that the left one is non-zero by our hypothesis. This fact leads to a contradiction. So $\ell$ is the maximum number of periodic orbits of (1) in $\mathscr{U}$.

Let us prove their hyperbolicity. Fix one periodic orbit $\gamma=\{(x(t), y(t)), t \in$ $[0, T]\} \subset \mathscr{U}$, where $T$ is its period. Remember that $\gamma \cap\{D=0\}=\emptyset$. In order to 
study its hyperbolicity and stability we have to compute $\int_{0}^{T} \operatorname{div} X(x(t), y(t)) d t$, and to prove that it is not zero. This fact follows by integrating the equality

$$
\operatorname{div} X=\frac{\partial P}{\partial x}+\frac{\partial Q}{\partial y}=\frac{\operatorname{div}(D X)}{D}-\frac{\frac{\partial D}{\partial x} P+\frac{\partial D}{\partial y} Q}{D}
$$

because the last term of the right hand side of the above equality coincides with $\frac{d}{d t} \ln |D(x(t), y(t))|$.

To apply the above theorem, we consider a function $D(x, y)$ of the form $|V(x, y)|^{m}$ where $V$ is a smooth function in two variables in $\mathbb{R}^{2}$ and $m$ is a real number.

Before giving the result for this particular choice of $V$ we introduce some more notation. Given an open subset $\mathscr{W}$ with smooth boundary and a smooth function $V: \mathscr{W} \rightarrow \mathbb{R}$ we denote by $\ell(\mathscr{W}, V)$ the sum of $\ell(\mathscr{U})$ where $\mathscr{U}$ ranges over all the connected components of $\mathscr{W} \backslash\{V=0\}$. Finally, we denote by $c(\mathscr{W}, V)$ the number of closed ovals of $\{V=0\}$ contained in $\mathscr{W}$. See Figure 1 for an illustration of these definitions.

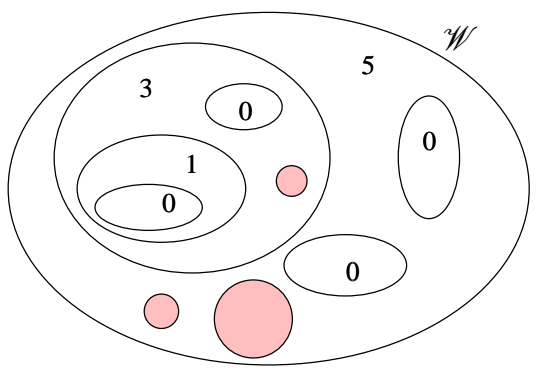

Fig. 1 Open set $\mathscr{W}$ with $\ell(\mathscr{W})=3$. The grey cercles are holes in $\mathscr{W}$ and the thick lines correspond to $\{V=0\}$. The numbers displayed are the values $\ell(\mathscr{U})$ for each connected component $\mathscr{U}$ of $\mathscr{W} \backslash\{V=0\}$. For this example $c(\mathscr{W}, V)=6$ and $\ell(\mathscr{W}, V)=9$.

Corollary 1. Assume that there exist a real number $s$ and an analytic function $V$ in $\mathbb{R}^{2}$ such that

$$
M_{s}:=\frac{\partial V}{\partial x} P+\frac{\partial V}{\partial y} Q+s\left(\frac{\partial P}{\partial x}+\frac{\partial Q}{\partial y}\right) V=\langle\nabla V, X\rangle+s V \operatorname{div}(X)
$$

does not change sign in an open region $\mathscr{W} \subset \mathbb{R}^{2}$ with regular boundary and vanishes only in a null measure Lebesgue set. Then the limit cycles of system (1) are either totally contained in $\mathscr{V}_{0}:=\{V=0\}$, or do not intersect $\mathscr{V}_{0}$.

Moreover the number of limit cycles contained in $\mathscr{V}_{0}$ is at most $c(\mathscr{W}, V)$ and the number $N$ of limit cycles that do not intersect $\mathscr{V}_{0}$ satisfies

$$
N \leq \begin{cases}\ell(\mathscr{W}) & \text { if } \quad s>0 \\ 0 & \text { if } \quad s=0 \\ \ell(\mathscr{W}, V) & \text { if } \quad s<0\end{cases}
$$

Furthermore for any $s \neq 0$ the limit cycles of this second type are hyperbolic. 
Proof. First observe that since $M_{S}$ does not change sign we have that on the analytic curves $\mathscr{V}_{0},\langle\nabla V, X\rangle$ does not change sign. Therefore these curves are either solutions of (1) or curves crossed by the flow generated by (1) in just one direction. Hence all limit cycles in $\mathscr{W}$ are either contained in the connected components of $\mathscr{W} \backslash \mathscr{V}_{0}$ or in $\mathscr{V}_{0}$. This fact implies the first assertions of the Theorem. In order to bound the number of limit cycles of (1) we apply the extended Bendixson-Dulac Theorem to each one of the connected components $\mathscr{U}$ of $\mathscr{W} \backslash \mathscr{V}_{0}$. The fact that when $D=|V|^{m}$,

$$
\operatorname{div}(D X)=\langle\nabla D, X\rangle+D \operatorname{div}(X)=\operatorname{sign}(V) m|V|^{m-1}\left[\langle\nabla V, X\rangle+\frac{1}{m} V \operatorname{div}(X)\right],
$$

gives the theorem by taking $m=1 / s$. Observe that the difference between the cases $s>0$ and $s<0$ comes from the fact that in the first case the function $D$ is well defined in the whole plane. For the case $s=0$ the proof is easier because $M_{0}=$ $d V / d t=\langle\nabla V, X\rangle$.

The above corollary shows that the study of the functions

$$
M_{s}=\frac{\partial V}{\partial x} P+\frac{\partial V}{\partial y} Q+s\left(\frac{\partial P}{\partial x}+\frac{\partial Q}{\partial y}\right) V
$$

gives a tool for controlling the number of limit cycles of system (1). As we will see this approach turns out to be useful for many families of planar vector fields. This function is also often used in the quoted works of Cherkas and his coauthors.

\subsection{Some simple examples}

As paradigmatic examples we will give short and easy proofs of the non-existence of limit cycles for a generalization of the Lotka-Volterra system and of the uniqueness of the limit cycle of the van der Pol system. The first one is a folklore prove and the second one is given by Cherkas, see [11, p. 105]. We will prove also a more general non-existence result for Kolmogorov systems.

\subsubsection{Non-existence of limit cycles for some predator-prey systems}

Consider the following extension of the celebrated Lotka-Volterra system

$$
\dot{x}=x(a x+b y+c), \quad \dot{y}=y(d x+e y+f),
$$

where all the parameters are real numbers. It appears in most texts books of mathematical ecology. By uniqueness of solutions it is clear that if it has periodic orbits then they do not intersect the coordinate axes. By making the change of variables 
$x \rightarrow \pm x, y \rightarrow \pm y$, if necessary, we can restrict our attention to the first quadrant $\mathscr{U}$ and prove that the system has no periodic orbit in it. To do this consider the Dulac function $D(x, y)=x^{A} y^{B}$, where the real numbers $A$ and $B$ have to be determined. Then the function $M$ appearing in (2) is

$$
\begin{aligned}
M(x, y) & =\langle\nabla D(x, y), X(x, y)\rangle+D(x, y) \operatorname{div} X(x, y) \\
& =x^{A} y^{B}((a A+d B+2 a+d) x+(b A+e B+2 e+b) y+(c A+f B+c+f)) .
\end{aligned}
$$

When $a e-b d \neq 0$ we can solve the linear system obtained vanishing the coefficients of $x$ and $y$ with unknowns $A$ and $B$. Call the solution $A=\alpha$ and $B=\beta$. Then

$$
M(x, y)=\frac{a b f+c e d-a e f-a c e}{a e-b d} x^{\alpha} y^{\beta}:=R x^{\alpha} y^{\beta} .
$$

When $R \neq 0$ we can apply the Bendixson-Dulac Theorem and since $\mathscr{U}$ is simply connected $(\ell(\mathscr{U})=0)$ the system has no limit cycles. When $R=0$ then it $x^{\alpha} y^{\beta}$ is an integrating factor. Hence the system is integrable and its first integral is smooth in $\mathscr{U}$. Thus it can not have isolated periodic orbits, i.e. it has no limit cycles. This case includes the famous Lotka-Volterra system. Recall that it has a center in $\mathscr{U}$, surrounded by periodic orbits.

When $a e-b d=0$ then either the linear system $a x+b y+c=0, d x+e y+f=0$, with unknowns $x$ and $y$ has no solutions or its solutions are either the full plane or a whole line. In the first case the only critical points of system (4) are on the axes, so the system can not have periodic orbits. Otherwise it is either the trivial system $\dot{x}=0, \dot{y}=0$ or is a reparameterization of the simple system $\dot{x}=g x, \dot{y}=h y$, for some real numbers $g, h$, which clearly can not have periodic orbits either.

\subsubsection{Non-existence of limit cycles for a class of Kolmogorov systems}

Following [2] we give a non-existence criterion for a family of Kolmogorov systems. This result can be applied to the Gause-type systems considered in [30] or to the systems studied in [23].

Proposition 1. Consider the $\mathscr{C}^{1}$-system

$$
\dot{x}=x\left(g_{0}(x)+g_{1}(x) y\right), \quad \dot{y}=y\left(h_{0}(x)+h_{1}(x) y+h_{2}(x) y^{2}\right),
$$

for $x \geq 0, y \geq 0$. For any $\lambda \in \mathbb{R}$ define the functions:

$$
\begin{aligned}
& S_{\lambda}(x)=x\left[g_{0}^{\prime}(x) g_{1}(x)-g_{0}(x) g_{1}^{\prime}(x)\right]+\lambda h_{0}(x) g_{1}(x)-(1+\lambda) g_{0}(x) h_{1}(x), \\
& T_{\lambda}(x)=(2+\lambda) h_{2}(x) g_{1}(x) .
\end{aligned}
$$

Let $\mathscr{I} \subset \mathbb{R}^{+}$be an open interval. Assume that there exists a value of $\lambda$ such that $S_{\lambda}(x) T_{\lambda}(x) \geq 0$, for all $x \in \mathscr{I}$, and all its zeroes are isolated. Then system (5) does not have periodic orbits in the strip $\mathscr{U}=\mathscr{I} \times(0,+\infty)$. 
Proof. First, let us prove that if the system has a limit cycle then it can not intersect the set $\left\{(x, y) \mid x>0, g_{1}(x)=0\right\}$. This holds because if $\bar{x}>0$ is such that $g_{1}(\bar{x})=0$ then either $x=\bar{x}$ is an invariant line (i.e. also $g_{0}(\bar{x})=0$ ) or it is a line without contact, i.e $\left.\dot{x}\right|_{x=\bar{x}}=\bar{x} g_{0}(\bar{x}) \neq 0$. Hence, in the region where (5) can have periodic orbits we can always assume that $g_{1}$ does not vanish.

Consider now the family of Dulac functions $D(x, y)=y^{\lambda-1} Z(x)$, where $\lambda$ is given in the statement and $Z$ is an unknown function. Computing the function (2),

$$
\begin{aligned}
M(x, y)= & \operatorname{div}(D(x, y) X(x, y)) \\
=[ & \left(x g_{0}(x) Z(x)\right)^{\prime}+\lambda h_{0}(x) Z(x)+\left(\left(x g_{1}(x) Z(x)\right)^{\prime}+(\lambda+1) h_{1}(x) Z(x)\right) y \\
& \left.+\left((\lambda+2) h_{2}(x) Z(x)\right) y^{2}\right] y^{\lambda-1} .
\end{aligned}
$$

The solutions of the differential equation

$$
\left(x g_{1}(x) Z(x)\right)^{\prime}+(\lambda+1) h_{1}(x) Z(x)=0
$$

are

$$
Z_{x_{0}}(x)=\frac{\exp \left[-(\lambda+1) \int_{x_{0}}^{x} \frac{h_{1}(s)}{s g_{1}(s)} d s\right]}{x g_{1}(x)},
$$

where $x_{0}>0$ is an arbitrary contant. By taking the Dulac function $\widetilde{D}(x, y)=$ $y^{\lambda-1} Z_{x_{0}}(x)$, for a given $x_{0}>0$, and taking into account that $Z_{x_{0}}(x)$ satisfies (6), we obtain after some computations that

$$
M(x, y)=\frac{Z_{x_{0}}(x)}{g_{1}(x)}\left(S_{\lambda}(x)+T_{\lambda}(x) y^{2}\right) y^{\lambda-1} .
$$

Since on $\mathscr{I}, S_{\lambda}(x) T_{\lambda}(x) \geq 0$ we have proved that $M$ does not change sign in $\mathscr{U}=I \times(0,+\infty)$, which is simply connected, and vanishes on a set of zero Lebesgue measure given by some vertical straight lines. Hence, by the Bendixson-Dulac Theorem, the result is proved.

Observe that the function $S_{\lambda}(x)$ of Proposition 1 can also be written as

$$
S_{\lambda}(x)=g_{1}^{2}(x)\left[x\left(\frac{g_{0}(x)}{g_{1}(x)}\right)^{\prime}+\lambda \frac{h_{0}(x)}{g_{1}(x)}-(1+\lambda) \frac{g_{0}(x) h_{1}(x)}{g_{1}^{2}(x)}\right] .
$$

When $h_{1}(x) \equiv 0$, it essentially coincides with the one given in the non-existence criterion presented in [25, Thm 4.1]. 


\subsubsection{Uniqueness of the limit cycle for the van der Pol equation}

The second order van der Pol equation $\ddot{x}+\varepsilon\left(x^{2}-1\right) \dot{x}+x=0$, can be written as the planar system

$$
\dot{x}=y, \quad \dot{y}=-\varepsilon\left(x^{2}-1\right) y-x .
$$

Taking $V(x, y)=x^{2}+y^{2}-1$ we obtain that the associated function $M_{s}$ given in (3) is

$$
M_{s}(x, y)=-\varepsilon\left(x^{2}-1\right)\left(s x^{2}+(2+s) y^{2}-s\right) .
$$

Choosing $s=-2$ we get that $M_{-2}(x, y)=2 \varepsilon\left(x^{2}-1\right)^{2}$. So, for $\varepsilon \neq 0$, this function does not change sign and vanishes on two straight lines. Hence since, $\ell\left(\mathbb{R}^{2}, V\right)=$ 1 and $s<0$, by Corollary 1, we obtain the van der Pol system has at most one limit cycle, which when exists is hyperbolic, and lies outside the unit circle. This approach does not provide the existence of the limit cycle. The existence, for $\varepsilon \neq 0$, can be obtained studying the behavior of the flow at infinity.

\section{Control of the function $M_{S}$}

To apply the Dulac method to concrete examples the main difficulty is to find a suitable couple $s \in \mathbb{R}$ and $V$ and then control the sign of the function $M_{s}$ given in (3). Many times a good trick consists in trying to reduce the question to a one variable problem. This approach is developed in Subsection 2.1 following $[17,18]$.

Another point of view is to work in polar coordinates. Then the control of the corresponding function $M_{S}$ takes advantage of writing the functions as polynomials of the radial component with coefficients depending periodically on the angle. This approach has been followed in [19] and some results are presented in Subsection 2.2.

\subsection{The function $M_{S}$ is reduced to a one variable function}

\subsubsection{A first method}

Proposition 2. Consider a $\mathscr{C}^{1}$ system of the form

$$
\dot{x}=p_{0}(x)+p_{1}(x) y=P(x, y), \quad \dot{y}=q_{0}(x)+q_{1}(x) y+q_{2}(x) y^{2}=Q(x, y),
$$

with $p_{1}(x) \not \equiv 0$. For each $s \in \mathbb{R}$ and for each $n \in \mathbb{N}$ it is possible to associate to it a $(n+1)$-parameter family of functions $V_{n}\left(x, y ; c_{0}, c_{1}, \ldots, c_{n}\right):=V_{n}(x, y)$ of the form

$$
V_{n}(x, y)=v_{0}(x)+v_{1}(x) y+v_{2}(x) y^{2}+\cdots+v_{n}(x) y^{n},
$$

such that for each one of them the function (3), 


$$
M_{s, n}(x)=\left\langle\nabla V_{n},(P, Q)\right\rangle+s V_{n} \operatorname{div}(P, Q)
$$

is a function only of the $x$-variable.

Proof. Direct computations give

$$
\begin{aligned}
\left\langle\nabla V_{n},(\right. & P, Q)\rangle+s V_{n} \operatorname{div}(P, Q)= \\
& =\left[\left\{\left(s p_{1}^{\prime}+2 s q_{2}+n q_{2}\right) v_{n}+p_{1} v_{n}^{\prime}\right\} y^{n+1}+\mathscr{F}_{n}\left(v_{n}, v_{n-1}\right) y^{n}+\right. \\
& +\left\{\mathscr{F}_{n-1}\left(v_{n-1}, v_{n-2}\right)+n q_{0}(x) v_{n}(x)\right\} y^{n-1}+\cdots+ \\
& \left.+\left\{\mathscr{F}_{1}\left(v_{1}, v_{0}\right)+2 q_{0}(x) v_{2}(x)\right\} y+\left\{\left(s p_{0}^{\prime}+s q_{1}\right) v_{0}+p_{0} v_{0}^{\prime}+q_{0} v_{1}\right\}\right],
\end{aligned}
$$

where for each $j=1,2 \ldots, n$,

$$
\begin{aligned}
\mathscr{F}_{j}\left(v_{j}, v_{j-1}, v_{j}^{\prime}, v_{j-1}^{\prime}\right)= & \left(s p_{0}^{\prime}+s q_{1}+j q_{1}\right) v_{j}(x)+ \\
& \left.p_{0} v_{j}^{\prime}(x)+\left(s p_{1}^{\prime}+2 s q_{2}+(j-1) q_{2}\right)\right) v_{j-1}(x)+p_{1} v_{j-1}^{\prime}(x) .
\end{aligned}
$$

From the above expressions we can obtain a 1-parameter family of functions $v_{n}^{*}\left(x ; c_{n}\right):=v_{n}^{*}(x)$ such that the coefficient of $y^{n+1}$ vanishes, by solving a linear first order ordinary differential equation. Once we have $v_{n}^{*}$, from $\mathscr{F}_{n}\left(v_{n}^{*}, v_{n-1}\right)=0$ we get $v_{n-1}^{*}\left(x ; c_{n}, c_{n-1}\right):=v_{n-1}^{*}(x)$ and so on until we have found $v_{n}^{*}, v_{n-1}^{*}, \ldots, v_{0}^{*}$. Finally, we obtain

$$
\left\langle\nabla V_{n},(P, Q)\right\rangle+s V_{n} \operatorname{div}(P, Q)=\left[\left(s p_{0}^{\prime}+s q_{1}\right) v_{0}^{*}+p_{0}\left(v_{0}^{*}\right)^{\prime}+q_{0} v_{1}^{*}\right]=M_{s, n}(x),
$$

as we wanted to prove.

Corollary 2. Consider the generalized Liénard system

$$
\dot{x}=y-F(x):=P(x, y), \quad \dot{y}=-g(x):=Q(x, y) .
$$

If we take

$$
V_{2}(x, y)=\left(\frac{s(s+1)}{2}(F(x))^{2}+c_{1} s F(x)+2 G(x)+c_{0}\right)+\left(s F(x)+c_{1}\right) y+y^{2},
$$

where $G(x)=\int_{0}^{x} g(z) d z$, then

$$
\begin{aligned}
M_{s, 2}(x) & =\left\langle\nabla V_{2},(P, Q)\right\rangle+s V_{2} \operatorname{div}(P, Q) \\
& =-\frac{s(s+1)(s+2)}{2}(F(x))^{2} F^{\prime}(x)-s(s+1) c_{1} F(x) F^{\prime}(x) \\
& -(s+2) g(x) F(x)-2 s F^{\prime}(x) G(x)-s c_{0} F^{\prime}(x)-c_{1} g(x) .
\end{aligned}
$$

In particular, for $s=-1$ we have

$$
V_{2}(x, y)=\left(-c_{1} F(x)+2 G(x)+c_{0}\right)+\left(-F(x)+c_{1}\right) y+y^{2},
$$

and 


$$
M_{-1,2}(x)=2 F^{\prime}(x) G(x)+c_{0} F^{\prime}(x)-g(x) F(x)-c_{1} g(x) .
$$

As an application of the above results we prove here the uniqueness and hyperbolicity of the limit cycle of a Liénard system with a rational $F$. The uniqueness (without proving the hyperbolicity) for this system was already proved in [13]; see also [21]. Other applications are given in [17].

Proposition 3. The Liénard system

$$
\dot{x}=y-F(x), \quad \dot{y}=-x, \quad \text { with } \quad F(x)=\frac{x\left(1-c x^{2}\right)}{\left(1+c x^{2}\right)}
$$

and c a real positive constant, has at most one limit cycle. Furthermore, when it exists it is hyperbolic and unstable.

Proof. We apply Proposition 2 and Corollary 2 with $s=-1, n=2$ and $V(x, y)$ given by the rational function:

$$
V(x, y)=y^{2}-F(x) y+x^{2}
$$

Then

$$
M_{-1,2}(x)=\frac{-4 c x^{4}}{\left(1+c x^{2}\right)^{2}}<0 \text { for all } \quad x \neq 0 .
$$

The function $V(x, y)=0$ is a second degree polynomial in the variable $y$, with discriminant

$$
\Delta=x^{2}\left(\left(\frac{1-c x^{2}}{1+c x^{2}}\right)^{2}-4\right)=-\frac{x^{2}\left(c x^{2}+3\right)\left(3 c x^{2}+1\right)}{\left(1+c x^{2}\right)^{2}}<0 \quad \text { for } \quad \text { all } x \neq 0 .
$$

Hence the set $\{V=0\}$ reduces to the origin. Therefore $c\left(\mathbb{R}^{2}, V\right)=0$ and $\ell\left(\mathbb{R}^{2}, V\right)=$ 1. From Corollary B we conclude that system (8) has at most one limit cycle. The origin is the only critical point of this system and it is stable. Then, when the limit cycle exists it is hyperbolic and unstable.

\subsubsection{A second method}

Proposition 4. Consider a $\mathscr{C}^{1}$ system of the form

$$
\dot{x}=y=P(x, y), \quad \dot{y}=h_{0}(x)+h_{1}(x) y+h_{2}(x) y^{2}+y^{3}=Q(x, y),
$$

and fix a positive integer number $n$. There is a constructive procedure to obtain an $(n+1)$-th order linear differential equation

$$
y^{(n+1)}(x)+r_{n, n}(x) y^{(n)}(x)+\cdots+r_{n, 1}(x) y^{\prime}(x)+r_{n, 0}(x) y(x)=0,
$$

such that if $y(x)=v_{n}(x)$ is any of its solutions, we can define a function 


$$
V_{n}(x, y):=v_{n, 0}(x)+v_{n, 1}(x) y+v_{n, 2}(x) y^{2}+\cdots+v_{n, n}(x) y^{n},
$$

where $v_{n, n}(x)=v_{n}(x)$ and $v_{n, i}(x), i=0 \ldots n-1$, are obtained from given expressions involving $h_{i}(x), i=0,1,2, v_{n}(x)$ and their derivatives, such that the corresponding function $M_{s}$ given in (3) with $s=-n / 3$,

$$
M^{[n]}:=M_{-n / 3}=\left\langle\nabla V_{n},(P, Q)\right\rangle-\frac{n}{3} V_{n} \operatorname{div}(P, Q),
$$

is a function only of the $x$-variable.

Proof. For sake of simplicity we present the details of the proof only for the case $n=2$. Also, for sake of brevity and during this proof, when it appears a function of the $x$ variable that we do not want to specify we simply will write $*$.

Take $V_{2}(x, y)=v_{2,0}(x)+v_{2,1}(x) y+v_{2,2}(x) y^{2}:=v_{0}(x)+v_{1}(x) y+v_{2}(x) y^{2}$. Then

$$
\begin{aligned}
M^{[2]}= & \left\langle\nabla V_{n},(P, Q)\right\rangle-\frac{2}{3} \operatorname{div}(P, Q) V_{2}= \\
& \left(v_{2}^{\prime}(x)+\frac{2}{3} v_{2}(x) h_{2}(x)-v_{1}(x)\right) y^{3}+ \\
& \left(v_{1}^{\prime}(x)+\frac{4}{3} v_{2}(x) h_{1}(x)-\frac{1}{3} v_{1}(x) h_{2}(x)-2 v_{0}(x)\right) y^{2}+ \\
& \left(v_{0}^{\prime}(x)+\frac{1}{3} v_{1}(x) h_{1}(x)-\frac{4}{3} h_{2}(x) v_{0}(x)+2 v_{2}(x) h_{0}(x)\right) y+ \\
& \left(v_{1}(x) h_{0}(x)-\frac{2}{3} h_{1}(x) v_{0}(x)\right)
\end{aligned}
$$

By choosing the following expressions for $v_{0}$ and $v_{1}$

$$
\begin{aligned}
& v_{0}(x)=\frac{1}{2}\left(v_{1}^{\prime}(x)+\frac{4}{3} v_{2}(x) h_{1}(x)-\frac{1}{3} v_{1}(x) h_{2}(x)\right), \\
& v_{1}(x)=v_{2}^{\prime}(x)+\frac{2}{3} v_{2}(x) h_{2}(x),
\end{aligned}
$$

we get that the coefficients of $y^{2}$ and $y^{3}$ in $M^{[2]}$ vanish. Observe that $v_{1}(x)=$ $v_{2}^{\prime}(x)+* v_{2}(x)$ and that $v_{0}(x)=v_{2}^{\prime \prime}(x) / 2+* v_{2}^{\prime}(x)+* v_{2}(x)$. Hence if we substitute these equalities in the coefficient of $y$ in the expression of $M^{[2]}$ we get that it writes as $v_{2}^{\prime \prime \prime}(x) / 2+* v_{2}^{\prime \prime}(x)+* v_{2}^{\prime}(x)+* v_{2}(x)$. By imposing that this last expression be identically zero we get the linear ordinary differential equation (9) given in the statement of the lemma. Hence for these values of the functions $v_{i}, i=0,1,2$ the expression of $M^{[2]}$ is the function of one variable

$$
M^{[2]}(x)=v_{1}(x) h_{0}(x)-\frac{2}{3} h_{1}(x) v_{0}(x),
$$


as we wanted to prove.

The advantage of the above result is that for each it $n$ gives the freedom to choose any solution of a linear ordinary differential equation of order $n+1$. Then using it we have to prove that the corresponding $M^{[n]}$ does not change sign. This approach is used in [19] to study the particular case $a=e=0$ of the challenging question proposed in [12]:

Question. Consider the planar semi-homogeneous system

$$
\dot{x}=a x+b y, \quad \dot{y}=c x^{3}+d x^{2} y+e x y^{2}+f y^{3} .
$$

Is two its maximum number of limit cycles?

\subsection{Computations in polar coordinates}

To work in polar coordinates we will need the expression of $M_{S}$ in terms of the expression of the vector field (1) in polar coordinates,

$$
\begin{aligned}
\dot{r} & =R(r, \theta):=P(r \cos \theta, r \sin \theta) \cos \theta+Q(r \cos \theta, r \sin \theta) \sin \theta, \\
\dot{\theta} & =\Theta(r, \theta):=\frac{1}{r}(Q(r \cos \theta, r \sin \theta) \cos \theta-P(r \cos \theta, r \sin \theta) \sin \theta) .
\end{aligned}
$$

Lemma 1. Let $\dot{r}=R(r, \theta), \dot{\theta}=\Theta(r, \theta)$ be the expression (10) of system (1) in polar coordinates. Then the function $M_{s}$ given in (3) writes as

$$
\begin{aligned}
M_{s} & =\frac{\partial V}{\partial x} P+\frac{\partial V}{\partial y} Q+s\left(\frac{\partial P}{\partial x}+\frac{\partial Q}{\partial y}\right) V \\
& =\frac{\partial V}{\partial r} R+\frac{\partial V}{\partial \theta} \Theta+s\left(\frac{\partial R}{\partial r}+\frac{\partial \Theta}{\partial \theta}+\frac{R}{r}\right) V .
\end{aligned}
$$

Theorem 1. Consider the planar differential system (1),

$$
\dot{x}=P(x, y), \quad \dot{y}=Q(x, y),
$$

where $P$ and $Q$ are real polynomials of degree $n$ and $P(0,0)=Q(0,0)=0$. Define the polynomial

$$
p\left(r^{2}\right):=\frac{1}{2 \pi r} \int_{0}^{2 \pi} R(r, \theta) d \theta,
$$

where $R$ is given in (10) and set $w(r)=r^{2} p^{\prime}\left(r^{2}\right)$. Denote by $d$ the degree of $w$ and by $N^{+}$its number of non-negative roots. For each fixed $s \in \mathbb{R}$ consider the function 


$$
\begin{aligned}
M_{S}(r, \theta): & =R(r, \theta) w^{\prime}(r)+s\left(\frac{\partial R(r, \theta)}{\partial r}+\frac{\partial \Theta(r, \theta)}{\partial \theta}+\frac{R(r, \theta)}{r}\right) w(r) \\
& =: \sum_{i=1}^{n+d-1} m_{i}(s, \theta) r^{i}
\end{aligned}
$$

and, for any $i \geq 1$, let $\mu_{i}(s)$ be such that $\max _{\theta \in[0,2 \pi]} m_{i}(s, \theta) \leq \mu_{i}(s)$.

Then, if the polynomial

$$
\Phi_{s}(r):=\sum_{i=1}^{n+d-1} \mu_{i}(s) r^{i}
$$

is negative for all $r \in(0, \infty)$, system (1) has at most $N^{+}$limit cycles and all of them are hyperbolic.

Proof. We want to apply Corollary 1 to system (1) with $V(x, y)=w(r)$ and the value $s$ given in the statement of the Theorem. By hypothesis, we have

$$
M_{s}=M_{s}(r, \theta)=\sum_{i=1}^{n+d-1} m_{i}(s, \theta) r^{i} \leq \sum_{i=1}^{n+d-1} \mu_{i}(s) r^{i}=\Phi_{s}(r)<0
$$

for all $r \in(0, \infty)$. Notice that by the proof of Corollary 1 and because $M_{S}$ does no vanish there are no limit cycles in $\{w(r)=0\}$. Hence the maximum number of limit cycles is $\ell\left(\mathbb{R}^{2}\right)=0$ when $s \geq 0$ and $\ell\left(\mathbb{R}^{2}, w\right)$ if $s<0$. In fact notice that $\{w(r)=0\}$ is formed by the origin and $N^{+}-1$ disjoint concentric cercles. Therefore $\ell\left(\mathbb{R}^{2}, w\right)=N^{+}$and again by Corollary 1 the theorem follows.

Remark 1. (i) The choice of the function $V(x, y)=w(r)$ in Theorem 1 is motivated by the following fact: for the simple system that in polar coordinates writes as $\dot{r}=r p\left(r^{2}\right), \dot{\theta}=q\left(r^{2}\right)$, where $q$ is any arbitrary polynomial, it holds that the corresponding $M_{-1}$, given in (3), is always negative.

(ii) Following the proof of Corollary 1 it is not difficult to see that under the hypotheses of the Theorem 1, if the system has only the origin as a critical point then it has at least $N^{+}-2$ limit cycles, with alternating stability. The reason is that two consecutive circles of $\{w(r)=0\}$ always are the boundaries of positive or negative invariant regions.

We end this subsection with a concrete application of Theorem 1 to a 3-parameter family of planar vector fields. Consider the system

$$
\begin{aligned}
& \dot{x}=x\left(1-\left(x^{2}+y^{2}\right)\right)\left(2-\left(x^{2}+y^{2}\right)\right)-y+a x^{2} y+b x^{2} y^{2}, \\
& \dot{y}=x+y\left(1-\left(x^{2}+y^{2}\right)\right)\left(2-\left(x^{2}+y^{2}\right)\right)+c x y^{2} .
\end{aligned}
$$

We will prove that if $a, b$ and $c$ are such that

$$
\Psi_{a, b, c}(r):=-10+\frac{9}{4}(|a|+|c|)+\frac{9}{4}|b| r+(12+|a|+|c|) r^{2}+|b| r^{3}-4 r^{4}<0
$$


for all $r>0$, then system (11) has at most two (hyperbolic) limit cycles. Moreover, when they exist, one is included in the disc $\mathscr{D}:=\left\{x^{2}+y^{2} \leq 3 / 2\right\}$ and is stable and the other one is outside the disc and it is unstable.

To apply Theorem 2 we compute $p(s)=2-3 s+s^{2}$. Then taking $w(r)=$ $r^{2} p^{\prime}\left(r^{2}\right)=r^{2}\left(-3+2 r^{2}\right)$ and $s=-1$, we obtain

$$
\begin{aligned}
M_{-1}(r, \theta)= & \frac{1}{4}(-40+a(6 \sin (2 \theta)-3 \sin (4 \theta))+c(6 \sin (2 \theta)+3 \sin (4 \theta))) r^{4} \\
& +\frac{3}{8} b(2 \cos (\theta)-3 \cos (3 \theta)+\cos (5 \theta)) r^{5} \\
& +(12+a \sin (4 \theta)-c \sin (4 \theta)) r^{6}-\frac{b}{2}(-\cos (3 \theta)+\cos (5 \theta)) r^{7}-4 r^{8} .
\end{aligned}
$$

Hence, for the values of the parameters considered, we can prove that

$$
M_{-1}(r, \theta) \leq r^{4} \Psi_{a, b, c}(r)<0
$$

for all $r>0$. Thus we can apply Theorem 2 . Since $N^{+}=2$ we have proved that system (11) has at most two (hyperbolic) limit cycles.

For instance the condition on $\Psi_{a, b, c}$ holds for $a=1 / 8, b=1 / 15$ and $c=1 / 20$. Moreover for these parameters it is not difficult to prove, by using resultants and the Sturm's theorem, that the origin is the unique critical point, which is unstable. Finally, by studying the flow on $\left\{x^{2}+y^{2}=R^{2}\right\}$, for $R$ big enough, and on $\left\{x^{2}+y^{2}=\right.$ $3 / 2\}$, we prove the existence of both limit cycles.

\section{More applications}

This section contains an extension of a Massera's result extracted from [20] and a study of an extension of the van der Pol system introduced in [34].

\subsection{A generalization of a result of Massera}

Consider the generalized smooth second order Liénard equation.

$$
\ddot{x}+f(x) \dot{x}+g(x)=0,
$$

with $f$ and $g$ smooth functions. It can be written as the planar system

$$
\dot{x}=y, \quad \dot{y}=-f(x) y-g(x) .
$$

We define $G(x)=\int_{0}^{x} g(z) d z$.

Using once more the extended Bendixson-Dulac Theorem and its Corollary 1 we can prove the following result. 


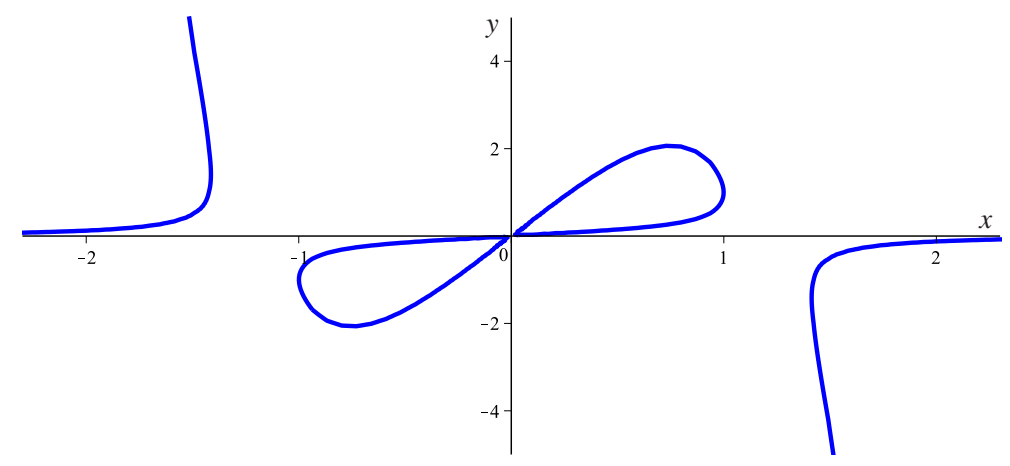

Fig. 2 Example of set $\{V=0\}$, under the hypotheses of Proposition 5.

Proposition 5. Let $\mathscr{W}=\mathscr{I} \times \mathbb{R}$ be a vertical strip of $\mathbb{R}^{2}$, where $\mathscr{I}$ is an open interval containing the origin. Assume that the functions $f$ and $g$ are of class $\mathscr{C}^{1}$, that $g$ only vanishes at the origin and that $f+2(f / g)^{\prime} G$ does not change sign on $\mathscr{I}$,vanishing only at $x=0$. Then system (12) has at most one periodic orbit which entirely lies in $\mathscr{W}$, and when it exists it is a hyperbolic limit cycle.

Proof. By taking $V(x, y)=y^{2}+(2 G f) y / g+2 G$ and $s=-1$ we can compute $M_{S}$ given in (3), obtaining that

$$
M_{-1}=\left(f+2 G(f / g)^{\prime}\right) y^{2} .
$$

Notice that $G / g$ and $(f / g)^{\prime} G$ are well-defined at the origin. By the hypotheses, $M_{-} 1$ does not change sign on $\mathscr{W}$ and $\left\{M_{-1}=0\right\}=\{x y=0\}$. Moreover this set does not contain periodic orbits. Hence we can apply Corollary 1 . Since $s<0$ we have to compute $\ell(\mathscr{W}, V)$. The function $V$ has degree 2 in $y$ and when $x=0$ the only point in $\mathscr{V}_{0}:=\{V=0\}$ is $(0,0)$. Therefore the set $\mathscr{V}_{0}$ has no oval surrounding the origin. Moreover, since the origin is the only critical point of the system and $\mathscr{V}_{0}$ is without contact by the flow of the system, then $\mathscr{V}_{0}$ does not contain ovals at all. In Figure 2 we illustrate a possible set $\mathscr{V}_{0}$, taking $f(x)=-4+x^{2}+x^{4}$ and $g(x)=x$. Then $V(x, y)=y^{2}+\left(-4+x^{2}+x^{4}\right) x y+x^{2}$ and $M_{-1}(x, y)=2\left(1+2 x^{2}\right) x^{2} y^{2}$. Hence, in general, all the connected regions of $\mathscr{W} \backslash \mathscr{V}_{0}$ are simply connected but one and $\ell(\mathscr{W}, V)=1$. Thus we have proved the uniqueness of the limit cycle.

We remark that Proposition 5 when $g(x)=x$, contains the following classical result, which was proved by Massera [28] and Sansone [32].

Massera's Theorem. Consider the Liénard differential system (12) with $g(x)=x$, $f(0)<0$ and $f^{\prime}(x) x>0$ if $x \neq 0$. Then system (12) has at most one limit cycle. 


\subsection{A generalization of van der Pol equation}

The system

$$
\dot{x}=y, \quad \dot{y}=-x+\left(b^{2}-x^{2}\right)\left(y+y^{3}\right),
$$

is introduced and studied in [34] as a generalization of the van der Pol equation. In the papers $[22,34]$ it is proved that it has at most one (hyperbolic) limit cycle and that it exists if and only if $b \in\left(0, b^{*}\right)$ for some $\left.0<b^{*}<\sqrt[6]{9 \pi^{2} / 16}\right) \approx 1.33$. This bifurcation value is refined in [16], proving that $b^{*} \in(0.79,0.817)$. In fact, numerically it can be seen that $b^{*} \approx 0.80629$. In this section we will prove the uniqueness of the limit cycle when $b \in(0,0.6]$ using a suitable Bendixson-Dulac function. This idea is developed in [16] where the authors prove, with the same method, the uniqueness and hyperbolicity of the limit cycle holds when $b \in(0,0.817)$ and its non-existence when $b \in[0.817, \infty)$.

To give an idea of how we have found the function $V$ and the value $s$ to find the function $M_{s}$ that we will use in our proof we first study again the van der Pol system. As we will see the main difficulty of this example is that the function $M_{s}$ is a function of two variables.

\subsubsection{The van der Pol equation (a second approach)}

The van der Pol equation studied in Subsection 1.2.3, after a rescaling of variables, is equivalent to the system

$$
\dot{x}=y, \quad \dot{y}=-x+\left(b^{2}-x^{2}\right) y .
$$

Arguing like in Section 2.1.1 it is natural to start considering functions of the form

$$
V(x, y)=v_{2} y^{2}+v_{1}(x) y+v_{0}(x),
$$

with $s=-1$. Then the corresponding $M_{-1}$ given in (3) is a polynomial of degree 2 in $y$, with coefficients being functions of $x$. In particular the coefficient of $y^{2}$ is

$$
v_{1}^{\prime}(x)+v_{2}\left(b^{2}-x^{2}\right) .
$$

Taking $v_{1}(x)=\left(x^{2}-3 b^{2}\right) v_{2} x / 3$ we get that this coefficient vanishes. Next, fixing $v_{2}=6$, and imposing to the coefficient of $y$ to be zero we obtain that $v_{0}(x)=6 x^{2}+c$, for any constant $c$. Finally, taking $c=b^{2}\left(3 b^{2}-4\right)$, we arrive to

$$
V(x, y)=6 y^{2}+2\left(x^{2}-3 b^{2}\right) x y+6 x^{2}+b^{2}\left(3 b^{2}-4\right) .
$$

Then

$$
M_{-1}(x, y)=4 x^{4}+b^{2}\left(3 b^{2}-4\right)\left(x^{2}-b^{2}\right) .
$$

It is easy to see that for $b \in(0,2 / \sqrt{3}) \approx(0,1.15), M_{-1}(x, y)>0$. Hence we can apply Corollary 1 . As $V(x, y)$ is quadratic in $y, V(x, y)=0$ has at most one oval, 
see Figure 3 for $b=1$. Hence $\ell\left(\mathbb{R}^{2}, V\right)=1$ and we have proved the uniqueness and hyperbolicity of the limit cycle when $b<2 / \sqrt{3}$. Recall that the proof given in Subsection 1.2.3 is simpler and valid for all values of the parameter. We have included this one as a motivation for the construction of the function $V(x, y)$ used to study system (13).

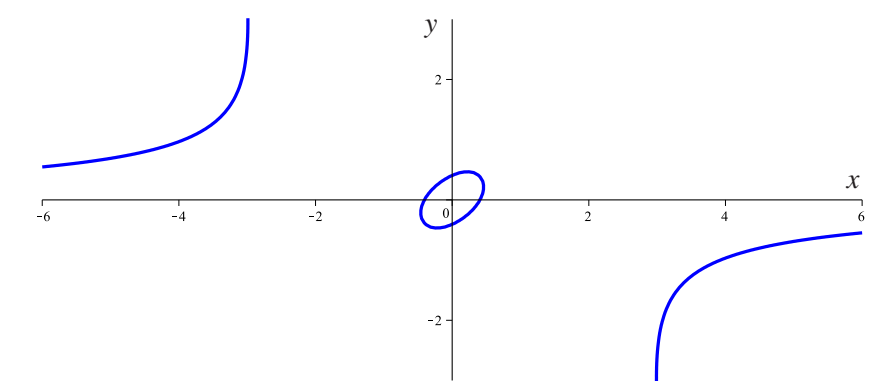

Fig. 3 The algebraic curve $V(x, y)=0$ with $b=1$.

\subsection{System (13) with $b \leq 0.6$}

By making some modifications to the function $V$ given by (15), we propose the following function $V$,

$$
\begin{aligned}
V(x, y)= & \left(2 x^{3}+6 b^{2}\left(1-b^{2}\right) x\right) y^{3}+6\left(1-b^{2}\right) y^{2}+2\left(x^{2}-3 b^{2}\right) x y \\
& +6\left(1-b^{2}\right) x^{2}+b^{2}\left(3 b^{2}-4\right)
\end{aligned}
$$

and again $s=-1$. Some computations give that

$$
\begin{aligned}
M_{-1}(x, y)= & 6\left(\left(2-3 b^{2}\right) x^{4} y^{2}-2 b^{2}\left(2-b^{2}\right) x^{3} y^{3}+\left(2-b^{2}\right) x^{2} y^{4}\right)+2\left(2-3 b^{2}\right) x^{4} \\
& -3 b^{2}\left(14-15 b^{2}\right) x^{2} y^{2}+12 b^{4}\left(2-b^{2}\right) x y^{3}-b^{2}\left(4-9 b^{2}\right) x^{2} \\
& +3 b^{4}\left(2-3 b^{2}\right) y^{2}+b^{4}\left(4-3 b^{2}\right) .
\end{aligned}
$$

Then we need to study the shape of the connected components of set $\mathbb{R}^{2} \backslash\{V=$ $0\}$ and the sign of $M_{-1}(x, y)$. It can be seen that the algebraic curve $V(x, y)=0$, with $V(x, y)$ given by (16), has no singular points and at most one closed oval for $b \in(0.0 .85]$. Moreover $M_{-1}(x, y)$ does not vanish for $b \in(0,0.651)$. Hence we can apply again Corollary 1 . Since $\ell\left(\mathbb{R}^{2}, V\right)=1$ we have proved the uniqueness and hyperbolicity of the limit cycle for $b \leq 0.6$ (in fact for $b \leq 0.651$ ).

The tools used to prove the above assertions are given in [16]. Among other methods the authors use discriminants, double discriminants, Sturm sequences and the study of the points at infinity of the algebraic curves. 
Acknowledgements The first author is supported by the MICIIN/FEDER grant number MTM200803437 and the Generalitat de Catalunya grant number 2009SGR410

\section{References}

1. M. J. Álvarez, A. Gasull, H. Giacomini, A new uniqueness criterion for the number of periodic orbits of Abel equations, J. Differential Equations 234, 161-176 (2007).

2. M. J. Álvarez, A. Gasull, R. Prohens, Limit cycles for two families of cubic systems, Nonlinear Anal. 75, 6402-6417 (2012).

3. M. Chamberland, A. Cima, A. Gasull, F. Mañosas. Characterizing asymptotic stability with Dulac functions, Discrete Contin. Dyn. Syst. 17, 59-76 (2007).

4. L. A. Cherkas, Estimation of the number of limit cycles of autonomous systems, Differential Equations 13, 529-547 (1977).

5. L. A. Cherkas, Dulac function for polynomial autonomous systems on a plane, Differential Equations 33, 692-701 (1997).

6. L. A. Cherkas, A. A. Grin', A second-degree polynomial Dulac function for a cubic system on the plane, Differential Equations 33, 1443-1445 (1997).

7. L. A. Cherkas, A. A. Grin', A Dulac function in a half-plane in the form of a polynomial of the second degree for a quadratic system, Differential Equations 34, 1346-1348 (1998).

8. L. A. Cherkas, A. A. Grin', K. R. Schneider, Dulac-Cherkas functions for generalized Liénard systems, Electron. J. Qual. Theory Differ. Equ. 35, 23 pp. (2011).

9. L. A. Cherkas, A. A. Grin', On the Dulac function for the Kukles system, Differerential Equations 46, 818-826 (2010).

10. L. A. Cherkas, A. A. Grin', A function of limit cycles of the second kind for autonomous functions on a cylinder, Differential Equations 47, 462-470 (2011).

11. C. Chicone, Ordinary differential equations with applications, Second edition, Texts in Applied Mathematics, 34. Springer, New York, 2006.

12. A. Cima, A. Gasull, F. Mañosas, Limit cycles for vector fields with homogeneous components, Appl. Math. (Warsaw) 24, 281-287 (1997).

13. R. Conti, Soluzioni periodiche dell'equazione di Liénard generalizatta. Esistenza ed unicità, Bolletino della Unione Matematica Italiana 3, 111-118 (1952).

14. F. Dumortier, J. Llibre and J.C. Artés, Qualitative theory of planar differential systems, UniversiText, Springer-Verlag, New York, 2006.

15. M. Fečkan, A generalization of Bendixson's criterion, Proc. Amer. Math. Soc. 129, 33953399 (2001).

16. J. D. García-Saldaña, A. Gasull, H. Giacomini, Bifurcation values for a family of planar vector fields of degree five, preprint 2012.

17. A. Gasull, H. Giacomini, A new criterion for controlling the number of limit cycles of some generalized Liénard equations, J. Differential Equations 185, 54-73 (2002).

18. A. Gasull, H. Giacomini, Upper bounds for the number of limit cycles through linear differential equations, Pacific J. Math. 226, 277-296 (2006).

19. A. Gasull, H. Giacomini, Upper bounds for the number of limit cycles of some planar polynomial differential systems, Discrete Contin. Dyn. Syst. 27, 217-229 (2010).

20. A. Gasull, H. Giacomini, J. Llibre, New criteria for the existence and non-existence of limit cycles in Liénard differential systems, Dyn. Syst. 24, 171-185 (2009).

21. A. Gasull, A. Guillamon, Non-existence, uniqueness of limit cycles and center problem in a system that includes predator-prey systems and generalized Liénard equations, Diff. Equations and Dynamical Systems 3, 345-366 (1995).

22. M. Han, T. Qian, Uniqueness of periodic solutions for certain second-order equations, Acta Math. Sin. (Engl. Ser.) 20, 247-254 (2004).

23. S.B. Hsu, T.W. Huang, Global stability for a class of predator-prey systems $\mathbf{5 5}, \mathbf{7 6 3 - 7 8 3}$ (1995). 
24. Yu. Ilyashenko, Centennial history of Hilbert's 16th problem, Bull. Amer. Math. Soc. (N.S.) 39, 301-354 (2002).

25. Y. Kuang, Global stability of Gause-type predator-prey systems, J. Math. Biol. 28, 463-474 (1990).

26. Y. Li, J. S. Muldowney, On Bendixson's criterion, J. Differential Equations 106, 27-39 (1993).

27. N. G. Lloyd, A note on the number of limit cycles in certain two-dimensional systems, J. London Math. Soc. (2) 20, 277-286 (1979).

28. J.L. Massera, Sur un théorème de G. Sansone sur l'équation di Liénard (French), Boll. Un. Mat. Ital. (3) 9, 367-369 (1954).

29. C. C. McCluskey, J. S. Muldowney, James S. Bendixson-Dulac criteria for difference equations, J. Dynam. Differential Equations 10, 567-575 (1998).

30. H.N. Moreira, On Liénard's equation and the uniqueness of limit cycles in predator-prey systems, J. Math. Biol. 28, 341-354 (1990).

31. L. M. Perko, "Differential equations and dynamical systems", Third edition. Texts in Applied Mathematics, 7. Springer-Verlag, New York, 2001.

32. G. Sansone, Soluzioni periodiche dell'equazione di Liénard. Calcolo del periodo (Italian), Univ. e Politecnico Torino. Rend. Sem. Mat. 10, 155-171 (1951).

33. G. Sansone, R. Conti, "Equazioni differenziali non lineari (Italian)", Edizioni Cremonese, Roma, 1956.

34. X. Wang, J. Jiang, P. Yan, Analysis of global bifurcation for a class of systems of degree five, J. Math. Anal. Appl. 222, 305-318 (1998).

35. G. Wilson, Hilbert's sixteenth problem, Topology 17, 53-73 (1978).

36. K. Yamato, An effective method of counting the number of limit cycles, Nagoya Math. J. 76, 35-114 (1979).

37. Yan Qian Ye \& others, "Theory of limit cycles", Translations of Mathematical Monographs 66. American Mathematical Society, Providence, RI, 1986.

38. Zhi Fen Zhang \& others, "Qualitative theory of differential equations", Translations of Mathematical Monographs 101. American Mathematical Society, Providence, RI, 1992. 